\title{
On a class of solvable difference equations generalizing an iteration process for calculating reciprocals
}

Stevo Stević1,2*

"Correspondence: sstevic@ptt.rs

'Mathematical Institute of the

Serbian Academy of Sciences and

Arts, Knez Mihailova 36/III, 11000

Beograd, Serbia

${ }^{2}$ Department of Medical Research,

China Medical University Hospital,

China Medical University, Taichung

40402, Taiwan, Republic of China

\begin{abstract}
The well-known first-order nonlinear difference equation

$$
y_{n+1}=2 y_{n}-x y_{n}^{2} \quad n \in \mathbb{N}_{0}
$$

naturally appeared in the problem of computing the reciprocal value of a given nonzero real number $x$. One of the interesting features of the difference equation is that it is solvable in closed form. We show that there is a class of theoretically solvable higher-order nonlinear difference equations that include the equation. We also show that some of these equations are also practically solvable.
\end{abstract}

MSC: 39A45

Keywords: Difference equation; Solvable equation; Theoretical solvability; Practical solvability; Closed-form formula

\section{Introduction}

Here we use the following standard notation: $\mathbb{N}, \mathbb{Z}, \mathbb{R}$, and $\mathbb{C}$ are the sets of natural, integer, real, and complex numbers, respectively, and $\mathbb{N}_{0}=\mathbb{N} \cup\{0\}$. If $p, q \in \mathbb{Z}, p \leq q$, then we use the notation $r=\overline{p, q}$ instead of writing $p \leq r \leq q, r \in \mathbb{Z}$.

In [10, p. 100], it is explained how the method of iteration can be used for approximating the values of a given function. Namely, if $f$ is a continuous function, then it is supposed to compute

$$
y=f(x)
$$

for a given value of variable $x$.

To compute the value of $f(x)$, it is suggested that relation (1) is written in an implicit form $F(x, y)=0$. An obvious way of writing relation (1) in an implicit form is by choosing $F(x, y)=y-f(x)$ (or $F(x, y)=f(x)-y$; for solving the equation, both choices are the same). However, depending on some properties of the function $f$, some other functions $F(x, y)$ can be also chosen. For example, if $f$ is invertible, then we can choose $F(x, y)=x-f^{-1}(y)$. If the

(c) The Author(s) 2021. This article is licensed under a Creative Commons Attribution 4.0 International License, which permits use, sharing, adaptation, distribution and reproduction in any medium or format, as long as you give appropriate credit to the original author(s) and the source, provide a link to the Creative Commons licence, and indicate if changes were made. The images or other third party material in this article are included in the article's Creative Commons licence, unless indicated otherwise in a credit line to the material. If material is not included in the article's Creative Commons licence and your intended use is not permitted by statutory regulation or exceeds the permitted use, you will need to obtain permission directly from the copyright holder. To view a copy of this licence, visit http://creativecommons.org/licenses/by/4.0/. 
range of $f$ does not contain the zero value, then, for example, we can also take $F(x, y)=$ $\frac{1}{f(x)}-\frac{1}{y}$, and so on. So, $F(x, y)$ can be chosen in various ways, and the choice depends on its usefulness in solving the problem of computation of $f(x)$. Two concrete examples of some suitable choices of function $F(x, y)$ are given below.

Such a chosen function $F(x, y)$ is a function of two variables, and the given $x$ in relation (1) (a fixed number therein) belongs to the domain of definition of the function.

Assume that the functions $F(x, y)$ and $F_{y}^{\prime}(x, y)$ are continuous and $F_{y}^{\prime}(x, y) \neq 0$. Let $y_{n}$ be an approximation of $y$. Then by the Langrage mean-value theorem we have

$$
F\left(x, y_{n}\right)=F\left(x, y_{n}\right)-F(x, y)=\left(y_{n}-y\right) F_{y}^{\prime}\left(x, \widehat{y}_{n}\right)
$$

where $\widehat{y}_{n}$ is a point between $y_{n}$ and $y$, and, consequently,

$$
y=y_{n}-\frac{F\left(x, y_{n}\right)}{F_{y}^{\prime}\left(x, \widehat{y}_{n}\right)} .
$$

It is natural to assume that $y_{n} \approx \widehat{y}_{n}$, so for computing $f(x)$, from (2) we obtain the following recursive relation:

$$
y_{n+1}=y_{n}-\frac{F\left(x, y_{n}\right)}{F_{y}^{\prime}\left(x, y_{n}\right)}, \quad n \in \mathbb{N}_{0}
$$

This is the Newton method, applied to $F$ as a function of $y$ (here $x$ is fixed).

Three nice examples for application of this method are given in [10]. Two of them are interesting for the present investigation. Hence we describe some relevant details.

The first example refers to computing reciprocals, that is, in this case, we have $f(x)=\frac{1}{x}$. Hence, for a given $x$, relation (1) becomes $y=\frac{1}{x}$.

To calculate it, choose the function $F(x, y)=x-\frac{1}{y}$. It is supposed to find $y$ for a given $x$ such that $F(x, y)=0$. Since $F_{y}^{\prime}(x, y)=\frac{1}{y^{2}}$, after some simple calculations, the recursive relation (3) becomes

$$
y_{n+1}=y_{n}\left(2-x y_{n}\right), \quad n \in \mathbb{N}_{0} .
$$

The recursive relation (4) appears in many problem books. For example, it is Problem 639.1 in the well-known problem book [9]. The reader might have seen it in the literature, but probably many are not aware of the above procedure, which leads to getting the relation. The problem in [9] is the following:

Problem 1 Let $x>0$, and let $\left(y_{n}\right)_{n \in \mathbb{N}_{0}}$ satisfy (4). Show that if $\min \left\{y_{0}, y_{1}\right\}>0$, then $y_{n}$ converges, and

$$
\lim _{n \rightarrow+\infty} y_{n}=\frac{1}{x}
$$

The problem is relatively simple and can be solved in several elementary ways. Some nice analyses related to the convergence of the sequence $y_{n}$ are given in [10]. It is interesting that (4) is a difference equation solvable in closed form. 
The hint given in [9] suggests consideration of the sequence $\frac{1}{x}-y_{n}$. From (4) we have

$$
\frac{1}{x}-y_{n+1}=\frac{1}{x}-2 y_{n}+x y_{n}^{2}, \quad n \in \mathbb{N}_{0}
$$

and, consequently,

$$
1-x y_{n+1}=\left(1-x y_{n}\right)^{2}, \quad n \in \mathbb{N}_{0}
$$

From (5) we easily obtain

$$
1-x y_{n}=\left(1-x y_{0}\right)^{2^{n}}, \quad n \in \mathbb{N}_{0}
$$

from which it follows that

$$
y_{n}=\frac{1-\left(1-x y_{0}\right)^{2^{n}}}{x}, \quad n \in \mathbb{N}_{0} .
$$

Formula (6) is a closed-form formula for general solution to equation (4) in the case $x \neq 0$. The case $x=0$ is very simple, since in this case, (4) defines a geometric progression with quotient equal to two, and hence $y_{n}=2^{n} y_{0}, n \in \mathbb{N}_{0}$. However, from the practical point of view, this case is not interesting, since for $x=0$, the function $\frac{1}{x}$ is not defined. By using formula (6) the long-term behavior of solutions to equation (6) can be easily described.

The second example in [10] refers to computing the square roots of positive numbers. This means that $f(x)=\sqrt{x}$ in (1). Further, the following function is chosen $F(x, y)=y^{2}-x$. Hence, in this case, equation (3) becomes

$$
y_{n+1}=y_{n}-\frac{y_{n}^{2}-x}{2 y_{n}}=\frac{1}{2}\left(y_{n}+\frac{x}{y_{n}}\right), \quad n \in \mathbb{N}_{0} .
$$

It is interesting that equation (7) is also solvable. Namely, we have

$$
y_{n+1} \pm \sqrt{x}=\frac{\left(y_{n} \pm \sqrt{x}\right)^{2}}{2 y_{n}}, \quad n \in \mathbb{N}_{0}
$$

from which it follows that

$$
\frac{y_{n+1}+\sqrt{x}}{y_{n+1}-\sqrt{x}}=\left(\frac{y_{n}+\sqrt{x}}{y_{n}-\sqrt{x}}\right)^{2}, \quad n \in \mathbb{N}_{0},
$$

and, consequently,

$$
\frac{y_{n}+\sqrt{x}}{y_{n}-\sqrt{x}}=\left(\frac{y_{0}+\sqrt{x}}{y_{0}-\sqrt{x}}\right)^{2^{n}}, \quad n \in \mathbb{N}_{0} .
$$

From (8) we obtain

$$
y_{n}=\sqrt{x} \frac{\left(\frac{y_{0}+\sqrt{x}}{y_{0}-\sqrt{x}}\right)^{2^{n}}+1}{\left(\frac{y_{0}+\sqrt{x}}{y_{0}-\sqrt{x}}\right)^{2^{n}}-1}, \quad n \in \mathbb{N}_{0} .
$$


Now we know that equation (7) is a particular case of a wider class of solvable nonlinear difference equations (see, e.g., [39] and the related references therein).

Solvable difference equations have attracted attention of researchers in the eighteenth and nineteenth centuries. Classical books on difference equations and related topics such as $[4,5,7,8,11,13-17,21-25]$ contain some information on the topic (see also the original sources $[3,6,18-20])$. In the last two decades, there has been some renewed interest in the topic (the reader can consult, e.g., [2, 26-42] and the related references therein).

Bearing in mind the above-mentioned facts related to equations (4) and (7), as well as some of our recent investigations on solvability of difference equations and systems, the following natural question arises.

Question 1 Is equation (4) also a particular case of a class of higher-order nonlinear difference equations solvable in closed form?

Here we give a positive answer to the question. We show that there is a class of theoretically solvable nonlinear difference equations that includes equation (4). We also show that some of them are practically solvable, presenting closed-form formulas for their general solutions.

Recall that we regard a difference equation as theoretically solvable if we know a form of its general solution, but some of the quantities appearing in the form of the general solution cannot be found in some suitable forms. For example, the homogeneous linear difference equations with constant coefficients

$$
y_{n+k}-a_{k-1} y_{n+k-1}-\cdots-a_{1} y_{n+1}-a_{0} y_{n}=0, \quad n \in \mathbb{N}_{0},
$$

where $k \in \mathbb{N}, a_{j} \in \mathbb{C}, j=\overline{0, k-1}$, are theoretically solvable, but some of them are not practically solvable, since the roots of the associated characteristic polynomials cannot be always found by radicals [1]. The roots can be always found by radicals if the degree of the polynomials is less than or equal to four [12].

\section{Main results}

In this section, we present our main results. First, we quote the following auxiliary result, which is employed for several times in the proofs of some of the main results [38].

Lemma 1 Let $m \in \mathbb{N}, l \in \mathbb{Z}, a_{j} \in \mathbb{R}, j=\overline{1, m-1}, a_{0} \in \mathbb{R} \backslash\{0\}$,

$$
\begin{aligned}
& \qquad R_{m}(s)=s^{m}-a_{m-1} s^{m-1}-\cdots-a_{1} s-a_{0}, \\
& R_{m}\left(s_{k}\right)=0, k=\overline{1, m}, s_{k} \neq s_{j}, k \neq j \text {, and let }\left(z_{n}\right)_{n \geq l-m} \text { be the solution to the difference equation } \\
& \quad z_{n}=a_{m-1} z_{n-1}+\cdots+a_{1} z_{n-m+1}+a_{0} z_{n-m} \\
& \text { for } n \geq l \text { such that } z_{j-m}=0, j=\overline{l, l+m-2}, z_{l-1}=1 . \text { Then } \\
& z_{n}=\sum_{k=1}^{m} \frac{s_{k}^{n+m-l}}{R_{m}^{\prime}\left(s_{k}\right)}, \quad n \geq l-m .
\end{aligned}
$$


Here we consider the higher-order nonlinear difference equation

$$
y_{n+k}=y_{n+l}+y_{n}+a y_{n+l} y_{n}, \quad n \in \mathbb{N}_{0},
$$

where $k \in \mathbb{N}, l \in \mathbb{N}_{0}, l<k, a \in \mathbb{C}$, and $y_{j} \in \mathbb{C}, j=\overline{0, k-1}$.

First note that there are two cases to be considered. The case when $a=0$, and the case when $a \neq 0$.

Case $a=0$. For $a=0$, equation (10) becomes the following particular case of equation (9):

$$
y_{n+k}=y_{n+l}+y_{n}, \quad n \in \mathbb{N}_{0} .
$$

The equation is well known and appears from time to time in the literature. For example, it has appeared recently in [35].

The characteristic polynomial associated with equation (11) is

$$
P_{k}(s)=s^{k}-s^{l}-1,
$$

and it can be certainly solved by radicals when $k \leq 4$. From this by a well-known theorem on the form of general solutions to homogeneous linear difference equations with constant coefficients $[4,13,14,21,22,24,25]$ it follows that equation (11) is practically solvable in this case. By the Abel-Ruffini theorem [1] the roots of a polynomial of degree strictly greater than four cannot be always found by radicals. Hence some of the polynomials in (12) for $k>5$ could be of this type.

Case $a \neq 0$. Since $a \neq 0$, we can multiply both sides of equation (10) by $a$. Then adding the unity to both sides of the obtained equation, we obtain the relation

$$
1+a y_{n+k}=1+a y_{n+l}+a y_{n}+a^{2} y_{n+l} y_{n}, \quad n \in \mathbb{N}_{0} .
$$

Now note that the last relation can be written as

$$
1+a y_{n+k}=\left(1+a y_{n+l}\right)\left(1+a y_{n}\right), \quad n \in \mathbb{N}_{0} .
$$

Relation (13) is very important for solvability of equation (10). Namely, by the substitution

$$
u_{n}=1+a y_{n}, \quad n \in \mathbb{N}_{0},
$$

equation (13) becomes

$$
u_{n+k}=u_{n+l} u_{n}, \quad n \in \mathbb{N}_{0} .
$$

If the initial values $u_{j}, j=\overline{0, k-1}$, are positive numbers, then a simple inductive argument shows that all the members of such a solution are also positive. Hence, in this case, it is possible to take the logarithm of both sides of equation (15) and use the substitution $v_{n}=\ln u_{n}, n \in \mathbb{N}_{0}$, by which the equation is transformed into equation (11). Hence equation (15) is theoretically solvable in the case of positive initial values. 
If some of the initial values are negative or complex numbers, then this method is not suitable for solving equation (15). However, there are some other methods for solving equations of this type, which are usually called product-type difference equations [33, 34].

If $k \leq 4$, then we can find the roots of the polynomial (12) by radicals, from which, together with the well-known theorem on the form of general solution to equation (9), we can find closed-form formulas for solutions to equation (11) and, consequently, closedform formulas for solutions to equation (15). Such formulas can be used in the following consequence of relation (14):

$$
y_{n}=\frac{u_{n}-1}{a}, \quad n \in \mathbb{N}_{0} .
$$

Hence the following particular cases of equation (15) are certainly practically solvable:

$$
\begin{array}{ll}
u_{n+2}=u_{n+1} u_{n}, & n \in \mathbb{N}_{0}, \\
u_{n+3}=u_{n+1} u_{n}, & n \in \mathbb{N}_{0}, \\
u_{n+3}=u_{n+2} u_{n}, & n \in \mathbb{N}_{0}, \\
u_{n+4}=u_{n+1} u_{n}, & n \in \mathbb{N}_{0}, \\
u_{n+4}=u_{n+2} u_{n}, & n \in \mathbb{N}_{0}, \\
u_{n+4}=u_{n+3} u_{n}, & n \in \mathbb{N}_{0} .
\end{array}
$$

From this it follows that to solve equation (10) in the cases $k \leq 4$, we should first solve equations (17)-(22) and then employ the obtained formulas for $u_{n}$ in relation (16). To solve the equations, we use some methods for solving product-type difference equations (see, e.g., $[30,33,34])$.

\subsection{Case $k=2, I=1$}

Let $\alpha_{1}=1$ and $\beta_{1}=1$. Then

$$
u_{n}=u_{n-1}^{\alpha_{1}} u_{n-2}^{\beta_{1}}, \quad n \geq 2,
$$

from which we obtain

$$
u_{n}=\left(u_{n-2} u_{n-3}\right)^{\alpha_{1}} u_{n-2}^{\beta_{1}}=u_{n-2}^{\alpha_{1}+\beta_{1}} u_{n-3}^{\alpha_{1}}=u_{n-2}^{\alpha_{2}} u_{n-3}^{\beta_{2}}, \quad n \geq 3
$$

where $\alpha_{2}=\alpha_{1}+\beta_{1}$ and $\beta_{2}=\alpha_{1}$.

By the same argument and induction we obtain that for each $m \in \mathbb{N} \backslash\{1\}$,

$$
\begin{aligned}
& u_{n}=u_{n-m}^{\alpha_{m}} u_{n-m-1}^{\beta_{m}}, \quad n \geq m+1, \\
& \alpha_{m}=\alpha_{m-1}+\beta_{m-1}, \quad \beta_{m}=\alpha_{m-1} .
\end{aligned}
$$

From (23) and (24) it easily follows that

$$
\begin{aligned}
& u_{n}=u_{1}^{\alpha_{n-1}} u_{0}^{\alpha_{n-2},}, \\
& \alpha_{n}=\alpha_{n-1}+\alpha_{n-2},
\end{aligned}
$$


and $\alpha_{1}=1$ and $\alpha_{2}=2$. Hence $\alpha_{n}=f_{n+1}\left(f_{n}\right.$ is the Fibonacci sequence [43]), and by (25) we obtain

$$
u_{n}=u_{1}^{f_{n}} u_{0}^{f_{n-1}}, \quad n \in \mathbb{N}_{0} .
$$

We obtain our first theorem by combining formulas (16) and (26).

Theorem 1 Let $k=2, l=1$, and $a \in \mathbb{C} \backslash\{0\}$. Then the formula

$$
y_{n}=\frac{\left(1+a y_{1}\right)^{f_{n}}\left(1+a y_{0}\right)^{f_{n-1}}-1}{a}, \quad n \in \mathbb{N}_{0}
$$

presents a general solution to equation (10) in this case.

\subsection{Case $k=3, I=1$}

Let $\alpha_{1}=1, \beta_{1}=1$, and $\gamma_{1}=0$. Then

$$
u_{n}=u_{n-2}^{\alpha_{1}} u_{n-3}^{\beta_{1}} u_{n-4}^{\gamma_{1}}, \quad n \geq 3,
$$

from which we obtain

$$
u_{n}=\left(u_{n-4} u_{n-5}\right)^{\alpha_{1}} u_{n-3}^{\beta_{1}} u_{n-4}^{\gamma_{1}}=u_{n-3}^{\beta_{1}} u_{n-4}^{\alpha_{1}+\gamma_{1}} u_{n-5}^{\alpha_{1}}=u_{n-3}^{\alpha_{2}} u_{n-4}^{\beta_{2}} u_{n-5}^{\gamma_{2}}, \quad n \geq 5
$$

where $\alpha_{2}=\beta_{1}, \beta_{2}=\alpha_{1}+\gamma_{1}$, and $\gamma_{2}=\alpha_{1}$.

By the same argument and induction we obtain that for each $m \in \mathbb{N} \backslash\{1\}$,

$$
\begin{aligned}
& u_{n}=u_{n-m-1}^{\alpha_{m}} u_{n-m-2}^{\beta_{m}} u_{n-m-3}^{\gamma_{m}}, \quad n \geq m+3, \\
& \alpha_{m}=\beta_{m-1}, \quad \beta_{m}=\alpha_{m-1}+\gamma_{m-1}, \quad \gamma_{m}=\alpha_{m-1} .
\end{aligned}
$$

From (29) it follows that

$$
\alpha_{n}=\alpha_{n-2}+\alpha_{n-3},
$$

and $\alpha_{0}=0, \alpha_{-1}=1, \alpha_{-2}=\alpha_{-3}=0, \alpha_{-4}=1((29)$ can be also used for $m \leq 1)$.

Using (28)-(30), we get

$$
u_{n}=u_{2}^{\alpha_{n-3}} u_{1}^{\beta_{n-3}} u_{0}^{\gamma_{n-3}}=u_{2}^{\alpha_{n-3}} u_{1}^{\alpha_{n-2}} u_{0}^{\alpha_{n-4}}, \quad n \in \mathbb{N}_{0} .
$$

We easily see that the roots $s_{j}, j=\overline{1,3}$, of the polynomial

$$
Q_{3}(s)=s^{3}-s-1=0,
$$

are distinct. From this and Lemma 1 it follows that

$$
\alpha_{n}=\sum_{j=1}^{3} \frac{s_{j}^{n+3}}{Q_{3}^{\prime}\left(s_{j}\right)}, \quad n \in \mathbb{Z},
$$

is the solution to (30) with $\alpha_{-3}=\alpha_{-2}=0$, and $\alpha_{-1}=1$.

From (16) and (31) we obtain our second theorem. 
Theorem 2 Let $k=3, l=1$, and $a \in \mathbb{C} \backslash\{0\}$. Then the formula

$$
y_{n}=\frac{\left(1+a y_{2}\right)^{\alpha_{n-3}}\left(1+a y_{1}\right)^{\alpha_{n-2}}\left(1+a y_{0}\right)^{\alpha_{n-4}}-1}{a}, \quad n \in \mathbb{N}_{0} \text {, }
$$

where $\alpha_{n}$ is defined in (33), presents a general solution to (10) in this case.

\subsection{Case $k=3, I=2$}

Let $\alpha_{1}=1, \beta_{1}=0$, and $\gamma_{1}=1$. Then

$$
u_{n}=u_{n-1}^{\alpha_{1}} u_{n-2}^{\beta_{1}} u_{n-3}^{\gamma_{1}}, \quad n \geq 3
$$

from which we obtain

$$
u_{n}=\left(u_{n-2} u_{n-4}\right)^{\alpha_{1}} u_{n-2}^{\beta_{1}} u_{n-3}^{\gamma_{1}}=u_{n-2}^{\alpha_{1}+\beta_{1}} u_{n-3}^{\gamma_{1}} u_{n-4}^{\alpha_{1}}=u_{n-2}^{\alpha_{2}} u_{n-3}^{\beta_{2}} u_{n-4}^{\gamma_{2}}, \quad n \geq 4,
$$

where $\alpha_{2}=\alpha_{1}+\beta_{1}, \beta_{2}=\gamma_{1}$, and $\gamma_{2}=\alpha_{1}$.

By the same argument and induction we obtain that for each $m \in \mathbb{N} \backslash\{1\}$

$$
\begin{aligned}
& u_{n}=u_{n-m}^{\alpha_{m}} u_{n-m-1}^{\beta_{m}} u_{n-m-2}^{\gamma_{m}}, \quad n \geq m+2, \\
& \alpha_{m}=\alpha_{m-1}+\beta_{m-1}, \quad \beta_{m}=\gamma_{m-1}, \quad \gamma_{m}=\alpha_{m-1} .
\end{aligned}
$$

From (36) we have

$$
\alpha_{n}=\alpha_{n-1}+\alpha_{n-3}
$$

and $\alpha_{0}=1, \alpha_{-1}=\alpha_{-2}=0, \alpha_{-3}=1, \alpha_{-4}=0$ ((36) can be also used for $\left.m \leq 1\right)$.

Formulas (35) and (36) yield

$$
u_{n}=u_{2}^{\alpha_{n-2}} u_{1}^{\beta_{n-2}} u_{0}^{\gamma_{n-2}}=u_{2}^{\alpha_{n-2}} u_{1}^{\alpha_{n-4}} u_{0}^{\alpha_{n-3}}, \quad n \in \mathbb{N}_{0} .
$$

It is not difficult to see that the roots $s_{j}, j=\overline{1,3}$, of the polynomial

$$
R_{3}(s)=s^{3}-s^{2}-1=0
$$

are different, from which, together with Lemma 1, it follows that

$$
\alpha_{n}=\sum_{j=1}^{3} \frac{s_{j}^{n+2}}{R_{3}^{\prime}\left(s_{j}\right)}, \quad n \in \mathbb{Z},
$$

is the solution to (37) with $\alpha_{-2}=\alpha_{-1}=0$, and $\alpha_{0}=1$.

Using (38) in (16) we get the following theorem.

Theorem 3 Let $k=3, l=2$, and $a \in \mathbb{C} \backslash\{0\}$. Then the formula

$$
y_{n}=\frac{\left(1+a y_{2}\right)^{\alpha_{n-2}}\left(1+a y_{1}\right)^{\alpha_{n-4}}\left(1+a y_{0}\right)^{\alpha_{n-3}}-1}{a}, \quad n \in \mathbb{N},
$$

where $\alpha_{n}$ is defined in (40), presents a general solution to (10) in this case. 


\subsection{Case $k=4, I=1$}

Let $\alpha_{1}=\beta_{1}=1$ and $\gamma_{1}=\delta_{1}=0$. Then

$$
u_{n}=u_{n-3}^{\alpha_{1}} u_{n-4}^{\beta_{1}} u_{n-5}^{\gamma_{1}} u_{n-6}^{\delta_{1}}, \quad n \geq 4
$$

from which we obtain

$$
u_{n}=\left(u_{n-6} u_{n-7}\right)^{\alpha_{1}} u_{n-4}^{\beta_{1}} u_{n-5}^{\gamma_{1}} u_{n-6}^{\delta_{1}}=u_{n-4}^{\beta_{1}} u_{n-5}^{\gamma_{1}} u_{n-6}^{\alpha_{1}+\delta_{1}} u_{n-7}^{\alpha_{1}}=u_{n-4}^{\alpha_{2}} u_{n-5}^{\beta_{2}} u_{n-6}^{\gamma_{2}} u_{n-7}^{\delta_{2}}
$$

for $n \geq 7$, where $\alpha_{2}=\beta_{1}, \beta_{2}=\gamma_{1}, \gamma_{2}=\alpha_{1}+\delta_{1}$, and $\delta_{2}=\alpha_{1}$.

By the same argument and induction we obtain that for each $m \in \mathbb{N} \backslash\{1\}$,

$$
\begin{aligned}
& u_{n}=u_{n-m-2}^{\alpha_{m}} u_{n-m-3}^{\beta_{m}} u_{n-m-4}^{\gamma_{m}} u_{n-m-5}^{\delta_{m}}, \quad n \geq m+5, \\
& \alpha_{m}=\beta_{m-1}, \quad \beta_{m}=\gamma_{m-1}, \quad \gamma_{m}=\alpha_{m-1}+\delta_{m-1}, \quad \delta_{m}=\alpha_{m-1} .
\end{aligned}
$$

From (43) we obtain

$$
\alpha_{n}=\alpha_{n-3}+\alpha_{n-4}
$$

and $\alpha_{0}=\alpha_{-1}=0, \alpha_{-2}=1, \alpha_{-3}=\alpha_{-4}=\alpha_{-5}=0, \alpha_{-6}=1$.

Using (42)-(44), we get

$$
u_{n}=u_{3}^{\alpha_{n-5}} u_{2}^{\beta_{n-5}} u_{1}^{\gamma_{n-5}} u_{0}^{\delta_{n-5}}=u_{3}^{\alpha_{n-5}} u_{2}^{\alpha_{n-4}} u_{1}^{\alpha_{n-3}} u_{0}^{\alpha_{n-6}}, \quad n \in \mathbb{N}_{0} .
$$

It is not difficult to see that the roots $s_{j}, j=\overline{1,4}$, of the polynomial

$$
R_{4}(s)=s^{4}-s-1=0
$$

are simple. From this by Lemma 1 it follows that

$$
\alpha_{n}=\sum_{j=1}^{4} \frac{s_{j}^{n+5}}{R_{4}^{\prime}\left(s_{j}\right)}, \quad n \in \mathbb{Z} .
$$

is the solution to (44) such that $\alpha_{-5}=\alpha_{-4}=\alpha_{-3}=0, \alpha_{-2}=1$.

The following result follows from (16) and (45).

Theorem 4 Let $k=4, l=1$, and $a \in \mathbb{C} \backslash\{0\}$. Then the formula

$$
y_{n}=\frac{\left(1+a y_{3}\right)^{\alpha_{n-5}}\left(1+a y_{2}\right)^{\alpha_{n-4}}\left(1+a y_{1}\right)^{\alpha_{n-3}}\left(1+a y_{0}\right)^{\alpha_{n-6}}-1}{a}, \quad n \in \mathbb{N}_{0} \text {, }
$$

where $\alpha_{n}$ is defined in (47), presents a general solution to (10) in this case.

\subsection{Case $k=4, I=2$}

In this case, we get (21), which is an equation with interlacing indices of second order [40, 42]. Thus the subsequences $u_{2 n}$ and $u_{2 n+1}$ are two solutions to equation (17). Employing Theorem 1, we obtain the following result. 
Theorem 5 Let $k=4, l=2$, and $a \in \mathbb{C} \backslash\{0\}$. Then the formulas

$$
\begin{aligned}
& y_{2 n}=\frac{\left(1+a y_{2}\right)^{f_{n}}\left(1+a y_{0}\right)^{f_{n-1}}-1}{a}, \quad n \in \mathbb{N}_{0}, \\
& y_{2 n+1}=\frac{\left(1+a y_{3}\right)^{f_{n}}\left(1+a y_{1}\right)^{f_{n-1}}-1}{a}, \quad n \in \mathbb{N}_{0},
\end{aligned}
$$

present a general solution to (10) in this case.

2.6 Case $k=4, I=3$

Let $\alpha_{1}=1, \beta_{1}=0, \gamma_{1}=0$, and $\delta_{1}=1$. Then

$$
u_{n}=u_{n-1}^{\alpha_{1}} u_{n-2}^{\beta_{1}} u_{n-3}^{\gamma_{1}} u_{n-4}^{\delta_{1}}, \quad n \geq 4
$$

from which we obtain

$$
u_{n}=\left(u_{n-2} u_{n-5}\right)^{\alpha_{1}} u_{n-2}^{\beta_{1}} u_{n-3}^{\gamma_{1}} u_{n-4}^{\delta_{1}}=u_{n-2}^{\alpha_{1}+\beta_{1}} u_{n-3}^{\gamma_{1}} u_{n-4}^{\delta_{1}} u_{n-5}^{\alpha_{1}}=u_{n-2}^{\alpha_{2}} u_{n-3}^{\beta_{2}} u_{n-4}^{\gamma_{2}} u_{n-5}^{\delta_{2}}
$$

for $n \geq 5$, where $\alpha_{2}=\alpha_{1}+\beta_{1}, \beta_{2}=\gamma_{1}, \gamma_{2}=\delta_{1}$, and $\delta_{2}=\alpha_{1}$.

By the same argument and induction we obtain that for each $m \in \mathbb{N} \backslash\{1\}$,

$$
\begin{aligned}
& u_{n}=u_{n-m}^{\alpha_{m}} u_{n-m-1}^{\beta_{m}} u_{n-m-2}^{\gamma_{m}} u_{n-m-3}^{\delta_{m}}, \quad n \geq m+3, \\
& \alpha_{m}=\alpha_{m-1}+\beta_{m-1}, \quad \beta_{m}=\gamma_{m-1}, \quad \gamma_{m}=\delta_{m-1}, \quad \delta_{m}=\alpha_{m-1} .
\end{aligned}
$$

From (50) we obtain

$$
\alpha_{n}=\alpha_{n-1}+\alpha_{n-4},
$$

and $\alpha_{0}=1, \alpha_{-1}=\alpha_{-2}=\alpha_{-3}=0, \alpha_{-4}=1, \alpha_{-5}=\alpha_{-6}=0$.

Relations (49) and (50) yield

$$
u_{n}=u_{3}^{\alpha_{n-3}} u_{2}^{\beta_{n-3}} u_{2}^{\gamma_{n-3}} u_{0}^{\delta_{n-3}}=u_{3}^{\alpha_{n-3}} u_{2}^{\alpha_{n-6}} u_{2}^{\alpha_{n-5}} u_{0}^{\alpha_{n-4}}, \quad n \in \mathbb{N}_{0} .
$$

It is not difficult to see that the roots $s_{j}, j=\overline{1,4}$, of the polynomial

$$
S_{4}(s)=s^{4}-s^{3}-1=0
$$

are simple. From this and Lemma 1 we have that

$$
\alpha_{n}=\sum_{j=1}^{4} \frac{s_{j}^{n+3}}{S_{4}^{\prime}\left(s_{j}\right)}, \quad n \in \mathbb{Z}
$$

is the solution to (51) such that $\alpha_{-3}=\alpha_{-2}=\alpha_{-1}=0$, and $\alpha_{0}=1$. 
From (16) and (52) we get our next result.

Theorem 6 Let $k=4, l=3$, and $a \in \mathbb{C} \backslash\{0\}$. Then the formula

$$
y_{n}=\frac{\left(1+a y_{3}\right)^{\alpha_{n-3}}\left(1+a y_{2}\right)^{\alpha_{n-6}}\left(1+a y_{1}\right)^{\alpha_{n-5}}\left(1+a y_{0}\right)^{\alpha_{n-4}}-1}{a}, \quad n \in \mathbb{N}_{0},
$$

where $\alpha_{n}$ is defined in (54), presents a general solution to (10) in this case.

Besides the above-described six cases where equation (10) is solvable in closed form, there are some other ones. Here we present one.

\subsection{Case $I=0$}

If $l=0$, then (10) is an equation with interlacing indices [40, 42]. If

$$
y_{m}^{(i)}=y_{m k+i}
$$

for $m \in \mathbb{N}_{0}, i=\overline{0, k-1}$, then

$$
y_{m+1}^{(i)}=2 y_{m}^{(i)}+a\left(y_{m}^{(i)}\right)^{2}, \quad m \in \mathbb{N}_{0}
$$

for each $i \in\{0, \ldots, k-1\}$, implying that $\left(y_{m}^{(i)}\right)_{m \in \mathbb{N}_{0}}, i=\overline{0, k-1}$, are solutions to (10) with $k=1$. Thus the solution to (10) consists of $k$ solutions to the equation with $k=1$. So, it is of interest to find a general solution in this case.

2.8 Case $k=1, I=0$

Since $k=1$, it follows that

$$
1+a y_{n+1}=\left(1+a y_{n}\right)^{2}, \quad n \in \mathbb{N}_{0}
$$

that is, $u_{n+1}=u_{n}^{2}, n \in \mathbb{N}_{0}$, and, consequently,

$$
u_{n}=u_{0}^{2^{n}}, \quad n \in \mathbb{N}_{0}
$$

Combining (16) and (57), we obtain the following theorem.

Theorem 7 Let $k=1, l=0$, and $a \in \mathbb{C} \backslash\{0\}$. Then the formula

$$
y_{n}=\frac{\left(1+a y_{0}\right)^{2^{n}}-1}{a}, \quad n \in \mathbb{N}_{0} \text {, }
$$

presents a general solution to (10) in this case.

2.9 Case $k \in \mathbb{N}, l=0$

From Theorem 7 we obtain the following result in the general case. 
Theorem 8 Let $k \in \mathbb{N}, l=0$, and $a \in \mathbb{C} \backslash\{0\}$. Then the formulas

$$
\begin{aligned}
& y_{m k}=\frac{\left(1+a y_{0}\right)^{2^{m}}-1}{a}, \quad m \in \mathbb{N}_{0}, \\
& y_{m k+1}=\frac{\left(1+a y_{1}\right)^{2^{m}}-1}{a}, \quad m \in \mathbb{N}_{0}, \\
& \vdots \\
& y_{m k+k-1}=\frac{\left(1+a y_{k-1}\right)^{2^{m}}-1}{a}, \quad m \in \mathbb{N}_{0},
\end{aligned}
$$

present a general solution to (10) in this case.

Remark 1 The roots of polynomials (32), (39), (46), and (53) can be found by radicals in some routine ways [12]. Hence we omit the calculations and leave them to the reader as simple exercises.

\subsection{A brief overview of some classes of solvable difference equations and the place of equation (10) in the classes}

The first nontrivial class of solvable difference equations appearing in the literature was (9) (the homogeneous linear with constant coefficients). The equations of small orders have been essentially solved for the first time by de Moivre [6-8] by using generating functions, whereas the equations of any order were solved by Bernoulli [3] by looking for solutions in the form $y_{n}=\lambda^{n}$. As an example, in [3], a closed-form formula for the Fibonacci sequence also appeared. Some formulas for solutions to the linear equations can be also found in Euler's book [11]. Lagrange [19] proposed the method of decomposition of the linear equations in linear factors and solved therein a first-order nonhomogeneous equation. He also proposed the method of undetermined coefficients for solving nonhomogeneous equations [18]. Another method for solving first-order nonhomogeneous equations was given by Laplace [20]. Therein he also solved, among other equations, some nonlinear ones, a few cyclic systems, and several partial difference equations. The paper indirectly shows that he also knew how to solve bilinear/fractional difference equations. Reduction of product-type difference equations to linear ones by using the logarithm has been known to mathematicians of the eighteenth century (see, e.g., [5], [16, p. 204]). We should say that the method is justified only for positive solutions to product-type difference equations. Some methods dealing with complex-valued solutions to product-type equations are described, for example, in [30,33, 34] (see also the related references therein). As we have shown here, equation (10) is transformed to a product-type difference equation, so it is a close relative of product-type equations, and, consequently, a bit farther relative of the homogeneous linear difference equations with constant coefficients.

Acknowledgements

Not applicable.

Funding

Not applicable.

Availability of data and materials Not applicable. 


\section{Competing interests}

The author declares that he has no competing interests.

\section{Authors' contributions}

The author has contributed solely to writing this paper. He read and approved the manuscript.

\section{Publisher's Note}

Springer Nature remains neutral with regard to jurisdictional claims in published maps and institutional affiliations.

\section{Received: 1 March 2021 Accepted: 3 April 2021 Published online: 13 April 2021}

\section{References}

1. Abel, N.H.: Mémoire sur les équations algébriques, ou l'on démontre l'impossibilité de la résolution de l'équation générale du cinquième degré Sylow, Ludwig; Lie, Sophus, Oeuvres Complètes de Niels Henrik Abel. (in French), I (2nd ed.), Grøndahl \& Søn, 28-33 (1881), 1824

2. Berg, L., Stević, S.: On some systems of difference equations. Appl. Math. Comput. 218, 1713-1718 (2011)

3. Bernoulli, $D$ : Observationes de seriebus quae formantur ex additione vel substractione quacunque terminorum se mutuo consequentium, ubi praesertim earundem insignis usus pro inveniendis radicum omnium aequationum algebraicarum ostenditur. Comment. Acad. Petropol. III 1728, 85-100 (1732) (in Latin)

4. Boole, G.: A Treatsie on the Calculus of Finite Differences, 3rd edn. Macmillan \& Co., London (1880)

5. Brunacci, V:: Corso di Matematica Sublime, Tomo I, Calcolo delle Differenze Finite e sue Applicazioni, Presso Pietro Allegrini, Firenze (1804) (in Italian)

6. de Moivre, A.: De Fractionibus algebraicis radicalitate immunibus ad fractiones simpliciores reducendis, deque summandis terminis quarumdam serierum aequali intervallo a se distantibus. Philos. Trans. 32, 162-178 (1722) (in Latin)

7. de Moivre, A.: Miscellanea Analytica de Seriebus et Quadraturis,. Tonson \& Watts, Londini (1730) (in Latin)

8. de Moivre, A.: The Doctrine of Chances, 3rd edn. Strand Publishing, London (1756)

9. Demidovich, B.P.: Sbornik Zadach i Uprazhnenii po Matematicheskomu Analizu, 8th edn. Nauka, Moskva (1972) (in Russian)

10. Demidovich, B.P., Maron, I.A.: Osnovy Vychislitelnoi Matematiki, 4th edn. Nauka, Moskva (1970) (in Russian)

11. Euler, L.: Introductio in Analysin Infinitorum, Tomus Primus, Lausannae (1748) (in Latin)

12. Faddeyev, D.K.: Lektsii po Algebre. Nauka, Moscow (1984) (in Russian)

13. Fort, T.: Finite Differences and Difference Equations in the Real Domain. Oxford University Press, London (1948)

14. Jordan, C.: Calculus of Finite Differences. Chelsea, New York (1956)

15. Krechmar, V.A.: A Problem Book in Algebra. Mir, Moscow (1974)

16. Lacroix, S.F.: Traité des Différences et des Séries. Duprat, Paris (1800) (in French)

17. Lacroix, S.F.: An Elementary Treatise on the Differential and Integral Calculus with an Appendix and Notes by J. Herschel. Smith, Cambridge (1816)

18. Lagrange, J.-L.: CEuvres, t. Il. Gauthier-Villars, Paris (1868) (in French)

19. Lagrange, J.-L.: Sur l'intégration d'une équation différentielle à différences finies, qui contient la théorie des suites récurrentes, Miscellanea Taurinensia. t. I, (1759), 33-42 (Lagrange Fuvres, I, 23-36, 1867). (in French)

20. Laplace, P.S.: Recherches sur l'intégration des équations différentielles aux différences finies, et sur leur usage dans la théorie des hasards. Mémoires de l' Académie Royale des Sciences de Paris 1773, t. VII, (1776) (Laplace CEuvres, VIII, 69-197, 1891) (in French)

21. Markoff, A.A.: Differenzenrechnung. Teubner, Leipzig (1896) (in German)

22. Milne-Thomson, L.M.: The Calculus of Finite Differences. Macmillan \& Co., London (1933)

23. Mitrinović, D.S., Adamović, D.D.: Nizovi i Redovi/Sequences and Series. Naučna Knjiga, Beograd (1980) (in Serbian)

24. Mitrinović, D.S., Kečkić, J.D.: Metodi Izračunavanja Konačnih Zbirova/Methods for Calculating Finite Sums. Naučna Knjiga, Beograd (1984) (in Serbian)

25. Nörlund, N.E.: Vorlesungen über Differenzenrechnung. Springer, Berlin (1924) (in German)

26. Papaschinopoulos, G., Schinas, C.J.: On the behavior of the solutions of a system of two nonlinear difference equations. Commun. Appl. Nonlinear Anal. 5(2), 47-59 (1998)

27. Papaschinopoulos, G., Schinas, C.J.: Invariants for systems of two nonlinear difference equations. Differ. Equ. Dyn. Syst. 7, 181-196 (1999)

28. Papaschinopoulos, G., Schinas, C.J.: Invariants and oscillation for systems of two nonlinear difference equations. Nonlinear Anal., Theory Methods Appl. 46, 967-978 (2001)

29. Papaschinopoulos, G., Stefanidou, G.: Asymptotic behavior of the solutions of a class of rational difference equations. Int. J. Difference Equ. 5(2), 233-249 (2010)

30. Rhouma, M.H.: The Fibonacci sequence modulo $\pi$, chaos and some rational recursive equations. J. Math. Anal. Appl. 310, 506-517 (2005)

31. Schinas, C.: Invariants for difference equations and systems of difference equations of rational form. J. Math. Anal. Appl. 216, 164-179 (1997)

32. Schinas, C.: Invariants for some difference equations. J. Math. Anal. Appl. 212, 281-291 (1997)

33. Stević, S.: First-order product-type systems of difference equations solvable in closed form. Electron. J. Differ. Equ. 2015, Article ID 308 (2015)

34. Stević, S.: Solvable product-type system of difference equations whose associated polynomial is of the fourth order. Electron. J. Qual. Theory Differ. Equ. 2017, Article ID 13 (2017)

35. Stević, S.: Solvability of a general class of two-dimensional hyperbolic-cotangent-type systems of difference equations. Adv. Differ. Equ. 2019, Article ID 294 (2019)

36. Stević, S.: Solvability of some classes of nonlinear first-order difference equations by invariants and generalized invariants. Electron. J. Qual. Theory Differ. Equ. 2019, Article ID 36 (2019) 
37. Stević, S.: Solving a class of non-autonomous difference equations by generalized invariants. Math. Methods Appl. Sci. 42, 6315-6338 (2019)

38. Stević, S.: New class of practically solvable systems of difference equations of hyperbolic-cotangent-type. Electron. J. Qual. Theory Differ. Equ. 2020, Article ID 89 (2020)

39. Stević, S.: A note on general solutions to a hyperbolic-cotangent class of systems of difference equations. Adv. Differ. Equ. 2020, Article ID 693 (2020)

40. Stević, S., Diblik, J., Iričanin, B., Šmarda, Z.: On some solvable difference equations and systems of difference equations. Abstr. Appl. Anal. 2012, Article ID 541761 (2012)

41. Stević, S., Iričanin, B., Kosmala, W.: More on a hyperbolic-cotangent class of difference equations. Math. Methods Appl. Sci. 42, 2974-2992 (2019)

42. Stević, S., Iričanin, B., Kosmala, W., Šmarda, Z.: Note on the bilinear difference equation with a delay. Math. Methods Appl. Sci. 41, 9349-9360 (2018)

43. Vorobiev, N.N.: Fibonacci Numbers. Birkhäuser, Basel (2002)

Submit your manuscript to a SpringerOpen ${ }^{\circ}$ journal and benefit from:

- Convenient online submission

- Rigorous peer review

Open access: articles freely available online

- High visibility within the field

- Retaining the copyright to your article

Submit your next manuscript at $\boldsymbol{\nabla}$ springeropen.com 